\title{
BMJ Open Study protocol for a single-blind randomised controlled trial to evaluate the clinical effects of an Integrated Qigong exercise intervention on freezing of gait in Parkinson's disease
}

\author{
Zhenlan Li, ${ }^{1}$ Jie Zhuang, ${ }^{1}$ Yan Jiang, ${ }^{1}$ Guiping Xiao, ${ }^{1}$ Kuncheng Jie, ${ }^{1}$ Tian Wang, \\ Wenhan Yin, ${ }^{1}$ Yu Zhang, ${ }^{2}$ Zhen Wang ${ }^{1}$
}

To cite: Li Z, Zhuang J, Jiang Y, et al. Study protocol for a single-blind randomised controlled trial to evaluate the clinical effects of an Integrated Qigong exercise intervention on freezing of gait in Parkinson's disease. BMJ Open 2019;9:e028869. doi:10.1136/ bmjopen-2018-028869

\section{- Prepublication history for} this paper is available online. To view these files, please visit the journal online (http://dx.doi. org/10.1136/bmjopen-2018028869).

Received 05 January 2019 Revised 04 August 2019 Accepted 05 August 2019

\section{Check for updates}

(C) Author(s) (or their employer(s)) 2019. Re-use permitted under CC BY-NC. No commercial re-use. See rights and permissions. Published by BMJ.

${ }^{1}$ School of Sport Science, School of Martial Arts, Shanghai University of Sport, Shanghai, China

${ }^{2}$ Department of Neurology, Xinhua Hospital Affiliated to Shanghai Jiaotong University School of Medicine, Shanghai, China

Correspondence to Professor Jie Zhuang; zhuangjiesh@163.com

Professor Zhen Wang; wangzhen@sus.edu.cn

\section{ABSTRACT}

Introduction Qigong exercise offers a potentially safe, low-cost and effective mind-body rehabilitative intervention for mitigating the problem of gait interruption among patients with Parkinson's disease (PD) who have frequent freezing of gait (FOG) episodes. However, its clinical effects have not been established. This paper describes the trial protocol of evaluating the clinical efficacy of a newly developed Integrated Qigong in improving gait among patients with PD who have FOG. Methods and analysis A single-blind randomised controlled trial is designed to compare Integrated Qigong and balance training with an attention control. Participants will be patients with mild to moderate PD who experience FOG and are recruited from local communities in Shanghai, China. Participants will be randomly allocated to one of the three groups: Integrated Qigong group, a balance exercise intervention group, or control group. The total number of participants will be 126, and masked assessments will be made at baseline, 12 weeks (end of intervention) and 12-week follow-up. Both Integrated Qigong group and balance training group will receive a group-based exercise intervention that meets three times per week, $60 \mathrm{~min}$ in duration, for 12 weeks. The control group will receive a 60 min weekly group session and monthly health education. The primary outcomes are gait parameters (stride length, gait velocity, stride time variability) and occurrence of FOG. The secondary outcomes are postural instability, walking disability, falling, fear of falling and quality of life.

Ethics and dissemination This study has been approved by the Ethics Committee of Shanghai University of Sport and registered at China Clinical Trial Registry. Participants will sign informed consent prior to the participation of the trial. The findings of the study will be published in peer-reviewed academic journals and disseminated to PD support groups, medical community and media. Trial registration number ChiCTR1800016570.

\section{INTRODUCTION}

Freezing of gait (FOG) is characterised by a sudden and brief episodic absence or marked reduction of forward progression. The

\section{Strengths and limitations of this study}

- The first study that combines commonly practised Qigong exercises into a single rehabilitative intervention aims at improving gait outcomes for patients with Parkinson's disease (PD) who have freezing of gait.

- The findings of the study will inform patients and healthcare providers of an alternative, potentially low-cost and safe, effective, and easily implementable exercise intervention for treating and managing gait interruption in patients with $\mathrm{PD}$.

- The patients will come from one geographic area which limits the generalisability.

syndrome is most common in patients with Parkinson's disease (PD). ${ }^{1}$ It is estimated that between $21 \%$ and $27 \%$ of early-stage patients report FOG episode with number increasing up to $80 \%$ among those in advanced stages..$^{2-4}$ Patients with FOG often show difficulty in turning, transferring, walking, and experience and inability to start or continue walking. As the disease progresses, patients with severe FOG develop postural instability and gait dysfunction, causing difficulty in managing daily life and frequent falls, which consequently impact on the quality of life. ${ }^{56}$

There are multidisciplinary approaches, including pharmacological and surgical-based treatment for managing FOG among people with PD. Current research evidence has shown, however, effectiveness of non-pharmacological and rehabilitation-based intervention, including physiotherapy, physical exercise and occupational therapy, for improving abnormal gait in patients with FOG. ${ }^{78}$ More research suggest that various alternative exercise interventions can ameliorate motor symptoms and improve gait. 


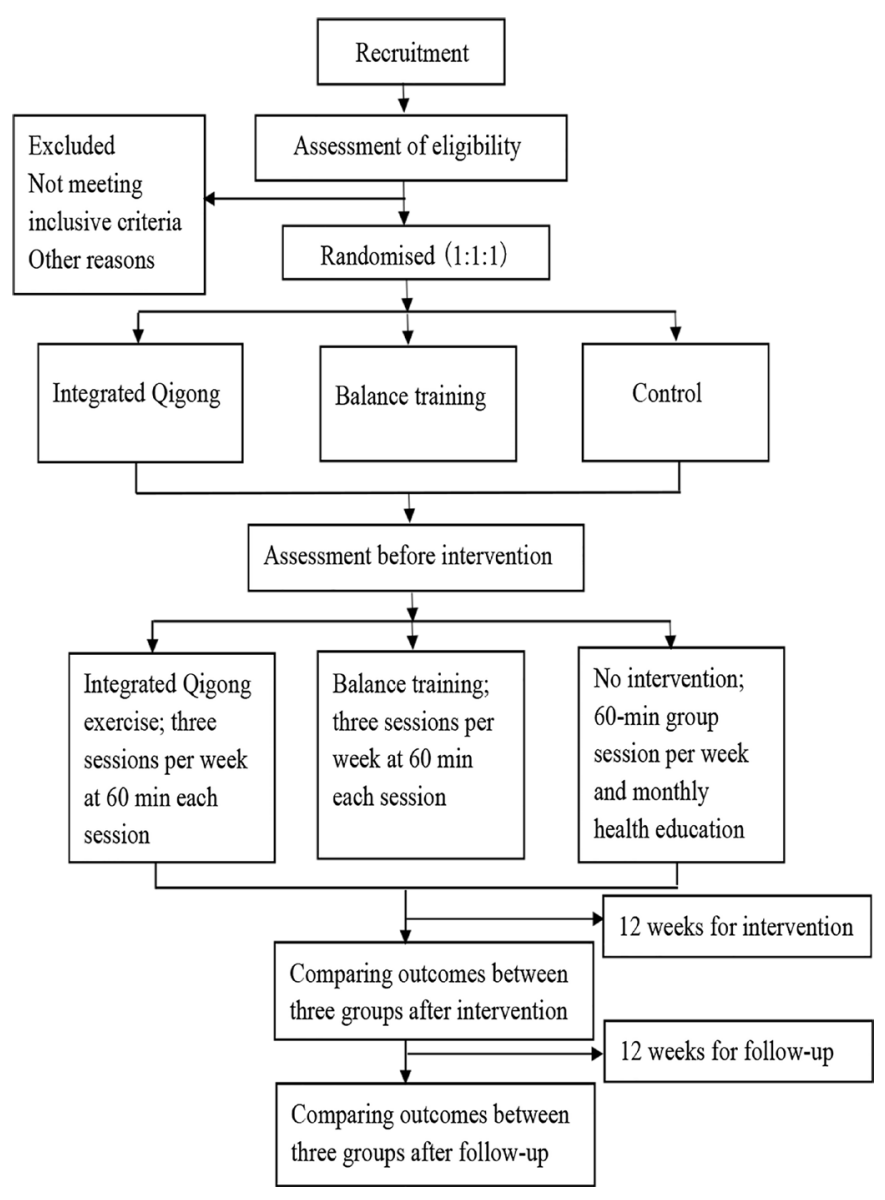

Figure 1 Flow diagram of study design.

Theses interventions include low-cost, non-equipment-dependent exercises such as tai chi, dance and yoga. ${ }^{9}$

In this study, we focus on Health Qigong exercise which is one of traditional Chinese exercises that incorporates meditative movements, breathing patterns and mental regulation. The exercises are performed in a slow, rhythmic, relaxed manner; features that are conducive to organs. ${ }^{1011}$ Growing evidence supports the health benefits of Health Qigong. A systematic review and meta-analysis indicates that older adults who practise Health Qigong improve balance and postural control and reduce fall risk among individuals with PD. ${ }^{12}$ Health Qigong also improves gait speed, stride length and leg movement ability. ${ }^{13} 14$

Qigong consists of various types, including Baduanjin, Liuzijue, Daoyin, 12-step Dao Yin Health Preservation Exercise, Yijinjing and Wuqinxi. Although each of them has its own training characteristics, they nevertheless share some common features. In general, Qigong integrates both static and dynamic exercises with a great emphasis on regulating breath, and exercising intrinsic control and mental intent. ${ }^{15}$ Qigong exercise is characterised by trunk rotation, bending and extending at waist, and movement of limbs both medial-laterally and anterior-posteriorly, all driven by the core of the body. ${ }^{14}{ }^{16}$ Like other techniques such as Tai Ji Quan, Qigong exercise also involves dynamic movements with intermittent stepping and turning.
Besides, exercise involves postural demanding movements such as single leg standing and chirographic and manipulative moving postures. ${ }^{17}{ }^{18}$ Furthermore, Qigong exercise requires regulating breathing by engaging in diaphragmatic breathing to expand lung capacity and control upright posture. ${ }^{19} 20$

To date, there has been little effort to evaluate the therapeutic effect of combing different types of Qigong exercise on gait in individuals with PD. This paper describes the trial protocol of a newly developed Integrated Qigong intervention that combines seven types of commonly exercised Qigong into a single rehabilitation programme for patients with mild to moderate PD who have FOG. Specifically, we aim to determine the therapeutic effect of the Integrated Qigong intervention on gait. We hypothesise that, compared with control group, both Integrated Qigong and conventional balance training intervention will be clinically more effective in improving the primary outcomes of gait.

\section{METHODS \\ Study design}

The study is designed as a prospective, single-blind randomised controlled trial (RCT).

\section{Design and procedures}

We will recruit participants from Shanghai, China, by means of the neurology department of the local hospital and TV programmes during the same recruitment period. To reduce potential expectation bias and confirm eligibility, a research assistant will make telephone contact with those referred by a neurologist and follow-up with interested participants. Participants will be informed that they will be randomly assigned to three groups. The Integrated Qigong exercise group will engage in training programme that combines seven types of commonly exercised Qigong. The balance training group will perform static and dynamic postural control training. The two intervention groups will receive the guidance of instructor, and the control group will receive weekly group session and monthly health education. The total intervention period will be 12 weeks, and will occur simultaneously for all participants. The primary and secondary outcomes will be assessed at baseline (preintervention), 12 weeks (end of intervention) and 12-week follow-up (see figure 1). Potential eligible participants who satisfy initial inclusion criteria will arrange a 2-hour visit at our research laboratory and register their personal information. All participants who meet the inclusion and exclusion criteria need to sign informed consent prior to the study.

\section{Participants}

Inclusion criteria

- Patients with PD diagnosed according to the clinical diagnostic of UK Brain Bank criteria.

- Age between 40 and 80 years old. 
- Hoehn and Yahr (H\&Y) scale of 1-3.

- Item 3 of the New Freezing of Gait Questionnaire (NFOGQ) scored $\geq 1$.

- Mini-Mental State Examination score >24.

- Ability to walk independently.

- Have experienced a fall over the past 6 months.

\section{Exclusion criteria}

- Participation in Qigong exercise during the last year.

- Other diseases that could interfere with conduct of the study protocol such as cardiopulmonary disease, liver and kidney disease, musculoskeletal dysfunction, or cancer.

- Severe cognitive, visual or auditory impairment.

- Unstable medication.

- Unstable deep brain stimulation.

\section{Sample size calculation}

A statistical analysis was conducted for sample size estimation using G*power V.3.1 (Franz Faul, Universitat Kiel, Germany). The study was powered to detect a betweengroup difference in primary gait outcome measures between Qigong exercise group and balance training group relative to the attention control group. Because there was no a priori hypothesis formulated between the two active interventions (Qigong exercise and balance training), the study was not powered on these two conditions. Due to the lack of informed preliminary data and empirical evidence on the effects of Qigong exercise, we approximated the effect size in our power calculations using estimated sample from the published studies that compared Qigong exercise with a control condition on gait (ie, gait speed, FOG and mobility). ${ }^{13}{ }^{14}$ In general, these studies, with an intervention length ranging from 10 to 24 weeks, have shown a weak intervention effect, with effect sizes ranging from no effect to small to moderate effects. On the basis of these observations, a conservative approach was taken. Specially, we used a small effect size $\left(\eta^{2}=0.01\right)$ based on the partial eta squared estimate within the analysis of variance (ANOVA) framework. ${ }^{21}$ Our initial power estimates indicated that, in a mixed-effects repeated measures design with a between-subject factor (Integrated Qigong, balance training, control), and a within-subject factor (baseline, 12 weeks) and a correlation of 0.08 on the repeated measures, a sample size of 99 subjects would be required to achieve an $80 \%$ power at a 0.05 error rate. To be more conservative, we estimated the sample sizes based on a range of small sizes $(f=0.08, f=0.10, f=0.12)$. The sample sizes generated from these calculations were averaged to arrive at a total of 107 subjects. With an anticipated $15 \%$ attrition rate, a final enrolment number of 126 (42 in each group) was set for the study to detect a difference in gait outcomes between Integrated Qigong and balance training relative to the attention control group.

\section{Randomisation and blinding}

The study design will use a single-blind RCT. Two trained assessors will be blinded to group allocation and will not participate in the intervention. We will apply the stratified random sampling method by stage of the disease ( $\mathrm{H} \& \mathrm{Y}$ stage). Using computer-generated random number sequencing (STATA V.12.0, StataCorp, Texas, USA) in a ratio of 1:1:1, a random number will be placed in a sealed envelope, and participants will be allocated randomly to Integrated Qigong group, balance training group, or control group by extracting the random number from the envelope. Participants will be reassessed for baseline measurements on another day. This study is not amenable to blinding to participants of their designated experimental groups because the interventional exercises they perform will reveal their group allocation.

\section{Intervention}

The participants in all groups will follow their regular medication scheme during the study period. Both Integrated Qigong group and balance training group will receive group-based exercise intervention at the sports science laboratory of Shanghai University of Sport. The group size is five to seven people in order to provide sufficient instructional attention to each participant. Two trained instructors will guide the participants to perform the exercises of their own group. The two interventional groups will perform threeweekly sessions of $60 \mathrm{~min}$ per session for 12 consecutive weeks. Each session will consist of $10 \mathrm{~min}$ of warm-up, $40 \mathrm{~min}$ of core exercises, $5 \mathrm{~min}$ of break intervals and 5 min of cool-down. The heart rates of the participants will be monitored by Polar-team² (Polar Electro, Finland) during training in order to progressively control the intensity. Participants in the interventional groups will be required to not perform any additional in-home exercises throughout the 12 weeks of training.

\section{Integrated Qigong exercise group}

The Integrated Qigong protocol will consist of 12 forms of exercise, with selected movements from the Health Qigong exercise guidelines organised and compiled by the China Qigong Management Center. The Integrated Qigong exercise will emphasise dynamic postural control and body weight shift stepping with lateral-medial and anterior-posterior movements, body symmetry pulling across up-down and left-right axes, and hand-eye coordination movement. The 12 forms of exercise have been previously documented $^{22}$ and can be seen in figure 2 .

During the initial 2-3 weeks, training will mainly emphasise learning and practising two or three forms through multiple repetitions along with review of previously learnt movements. The practice in each session concentrates on upper and lower limbs in place, trunk rotation, as well as stepping in different directions (ie, forward, backward, sideways, diagonal). Participants will be requested to perform personalised movement requirements based on functional level. The range of motion (ROM) of each movement will be reduced for participants with rigidity. The pace of movement will be decreased for participants with bradykinesia. For participants experiencing FOG episode during training, they will be instructed to 

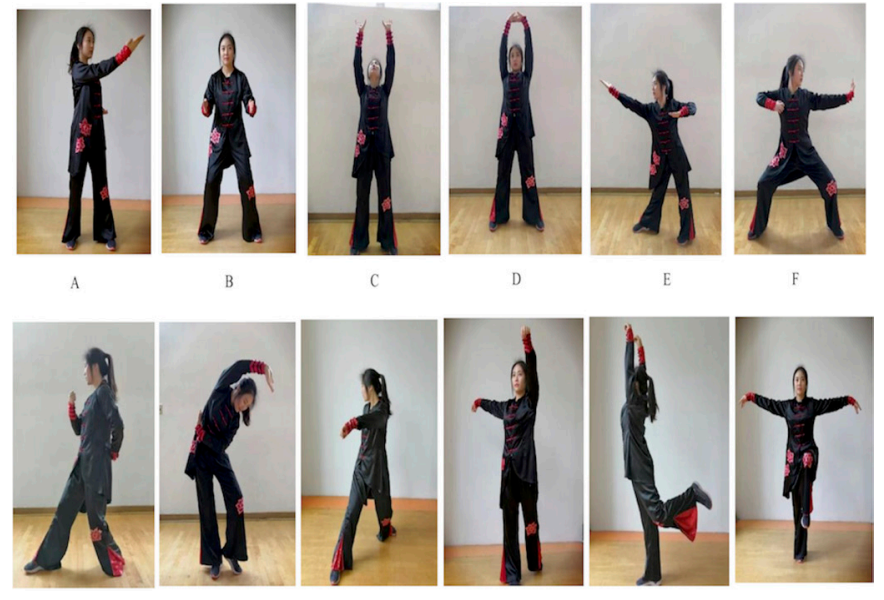

Figure 2 Twelve forms of Integrated Qigong exercise. (A) Form 1: Xu exercise. (B) Form 2: Chui exercise. (C) Form 3: Raising the tiger's paws. (D) Form 4: Holding the hands high with palms up to regulate the internal organ. (E) Form 5: Drawing a bow. (F) Form 6: Posing as an archer shooting both left and right handed. (G) Form 7: Pulling nine cows by their tails. (H) Form 8: Rub. backbone. (I) Form 9: Swaying like a bear. (J) Form 10: Picking fruit. (K) Form 11: Golden rooster heralds the dawn. (L) Form 12: Flying like a bird.

perform transferring and stepping while maintaining postural stability. The intensity, difficulty of movement, time and frequency will be adjusted to the demands of each participant. The later weeks will concentrate on improving balance, locomotion and action consistency. Participants will practise each movement with six repetitions, and natural breathing will be incorporated into the movement routine. Participants will be guided to perform the entire range of movement in which they feel safe.

\section{Balance training group}

Each training session will start with a 10 min warm-up consisting of breathing exercise, slow walking and rangeof-motion exercises. The $40 \mathrm{~min}$ balance training will include a short resting time, and the training programme will consist of the following: (1) static balance training: standing on unstable surfaces to maintain postural control and progression to weight shifting; (2) dynamic balance training: postural control in standing position while adding upper limb and trunk movements; (3) balance strategy exercise: focus on hip strategy while maintaining ankle strategy and stepping strategy under interference in different directions; (4) adaptation of varying base of support, and standing in a narrow space and on uneven surface ${ }^{23}$; and (5) walk integrated balance training: walk in a straight line, walk on a soft blanket and sideways. The training will progress from simple to complex, static to dynamic, low to high centre of gravity, wide to narrow the base of support, and will continue to raise challenges in regard to flexibility, stability and range of movement. The end of training will include a 5 min cooldown session of limb ROM movements, sustained stretching and relaxing.

\section{Control group}

The control group will be instructed to maintain their formal lifestyle and not to engage in any other form of intensive training. The participants in the control group will join one 60 min group session per week, which will consist of a $30 \mathrm{~min}$ lecture, $20 \mathrm{~min}$ discussion and will be followed by a $10 \mathrm{~min}$ question and answer session. Participants in the control group will receive health education every 4 weeks over the 12-week interventional period. The health education will involve information for PD-related treatments and prevention such as modalities of exercise, regimens, fall prevention and nutrition. Participants will receive a brochure of health education and will have follow-up by telephone twice per month, which will involve discussion of physical activity, progression of disease, health status and psychological status.

All participants will maintain diaries to record their exercise and fall events every day throughout the trial, including both in the laboratory and at home. The participants in the Integrated Qigong exercise group and balance training group will perform the exercises at the 'on' stage in the morning.

\section{Outcome measures}

All measurements will be performed at baseline, 12 weeks (end of intervention) and 12 weeks following the completion of the intervention. The measurements will be conducted by two trained assessors and will be videotaped by a third assessor. All assessors will be blinded to the participant's group allocation and time of assessment.

\section{Participants' characteristics}

Demographic and health characteristics of participants will be collected at baseline to describe the sample, compare conditions and investigate characteristics associated with outcomes. These characteristics will include age, gender, education, age at disease onset, disease duration, cognition ability, health status, medication dose, resting blood pressure, body mass $\left(\mathrm{kg} / \mathrm{m}^{2}\right)$, height $(\mathrm{cm})$, family situation and physical performance. Blood pressure will be measured with the use of an automated device (Omron HealthCare). Body mass and height will be assessed with digital scales (weighing scale and metre). Physical performance will be measured by the scores from self-reported habitual physical activity scale (see table 1 ).

\section{Primary outcome assessment}

Spatiotemporal kinematic parameter is one approach to characterise FOG, particularly the increased cadence, decreased stride before FOG episode. ${ }^{24}$ Hence, we will describe spatiotemporal characteristics to better understand the relationship between gait and FOG. Gait parameters will be analysed using a $7 \mathrm{~m}$ long instrumented computerised walkway (GAITRite, CIR Systems, Franklin, $\mathrm{NJ}$ ), and will include stride length, gait velocity, cadence and stride time variability. Gait velocity is a crucial parameter for walking coordination and can be used as basic factor for the assessment of normal and pathological 
Table 1 Demographic and clinical characteristics of the study participants

\section{Integrated Qigong}

(n)

\section{Balance training}

(n)
Control

(n)

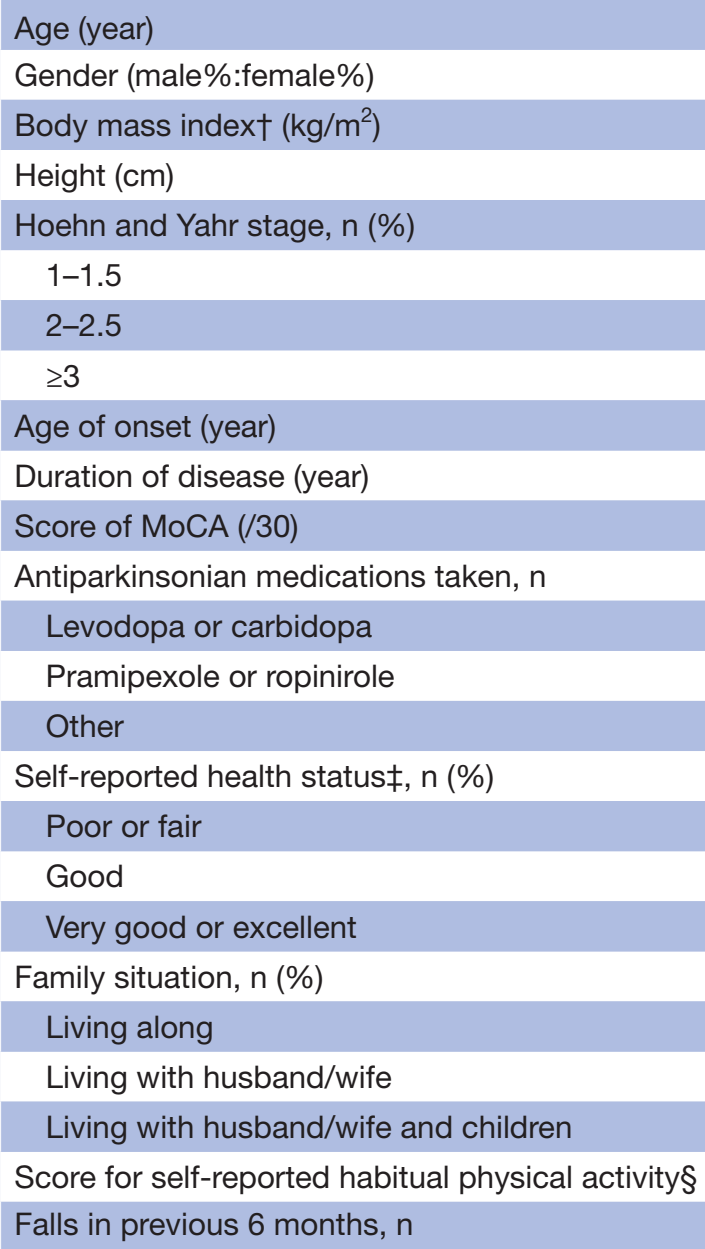

*Mean values (SD). The $\chi^{2}$ test is used for categorical variables, and one-way analysis of variance for continuous variables.

†The body mass index is the weight in kilograms divided by the square of the height in metres.

$\ddagger$ Self-reported health status included cardiovascular disease, lung disease, osteoporosis, arthritis, liver and kidney disease, low back pain and cancer; the number of conditions per participant ranged from 0 to 9.

$\S$ This is measured by the Physical Activity Scale for the Elderly, ${ }^{46}$ with higher scores indicating higher levels of habitual physical activity. MoCA, Montreal Cognitive Assessment.

gait. ${ }^{25}$ Gait variability is a valuable indicator of whole gait performance and can reflect gait disorders. Stride length and stride time variability are sensitive measures that relate to fall risk in older people. ${ }^{2627}$

NFOGQ is a reliable tool to detect freezing behaviour and assess effectiveness of intervention. It consists of the following three parts: (1) Part I (item 1, score is 0 or 1) detects the presence of FOG and distinguishes if patients are freezers or non-freezers if they have had a FOG experience during the past month. (2) Part II (items 2-6, score range from 0 to 9 ) assesses the severity of FOG according to its duration and frequency and its common appearance. Item 2 was added to rate the overall frequency of FOG regardless of the environment. (3) Part III (items $7-9$, score range from 0 to 9 ) assesses the influence of FOG on daily life, and the total score ranges from 0 to 28; higher scores reflect more severe FOG. ${ }^{28}$

\section{Secondary outcome assessment}

Functional Gait Assessment (FGA) is a measure of walking balance ability in older adults and individuals with PD and has shown good reliability and validity in the clinic. FGA includes the following conditions: forward, backward, with eyes closed, stepping over obstacles, changing gait speeds, with different head turns and a narrow base of support. The FGA includes 10 total items, with each item scored from 0 to 3. A higher total score reflects better balance and walking ability, with a maximum score of 30. ${ }^{29} 30$

Postural instability and gait disability will be evaluated by the Unified Parkinson's Disease Rating Scale Part III evaluation. It consists of rigidity measures, gait, tremor, hand/arm and leg movements (bradykinesia), speech and facial expressions. The modified H\&Y scale has also been used to evaluate disease severity. ${ }^{31}$ 
The balance performance will be measured by Mini-Balance Evaluation Systems Test, consisting of 14 items from four different balance control systems: anticipatory postural adjustments, reactive postural control, sensory orientation and dynamic gait. Each item is evaluated on a 3-point scale from 0 to 2 and the total score ranges from 0 to 28 , with a higher score indicating better balance performance. $^{32} 33$

Falling frequency will be reported at the baseline test, during the 12-week training period and at a 12-week follow-up. A blind assessor will record fall event based on the following definition of falling: 'a person unintentionally coming to rest on the ground or other lower level, not occurring as a result of a major intrinsic overwhelming hazard. ${ }^{34}$ The following approaches will be used to ascertain the fall event: (1) monthly collection of fall diary, (2) monthly phone interview, and (3) self-report from each assessment.

Falls and fear of falling will be measured with the 14-item Modified Falls Efficacy Scale questionnaire, which reflects self-perceived confidence to engage in activities of daily living without falling. Each item is scored on a 10 -point scale, with a minimum score of 0 indicating no confidence and a maximum score of 10 indicating full confidence (high falls efficacy) in performing the tasks without falling. The average score across all 14 items will be taken, with higher scores indicating greater falls efficacy. ${ }^{35} 36$

The quality of life will be assessed using the 39-item Parkinson's Disease Questionnaire. It is composed of 39 items grouped in eight domains: mobility, activities of daily living, emotional well-being, stigma, social support, cognition, communication and bodily discomfort. Each item ranges from 0 (never) to 4 (always). A summary index of eight domain scores ranges from 0 to 100 , with higher scores representing worse health-related quality of life. ${ }^{3738}$ The data collected are depicted in table 2 .

\section{Patient and public involvement}

Participants have not been involved in the study recruitment. The authors conceived the initial research questions and outcome measures, and modified according to the telephone interviews with patients and their guardians by a research assistant. In order to assure the safety and feasibility of the intervention, we invited six patients with mild to moderate PD to learn and practise the Integrated Qigong exercise before designing the RCT. Integrated Qigong movements were revised based on the exercise performance and feedback provided by the participants. The burden of the intervention will be assessed by patients and their advisors through face-to-face interviews before signing informed consent. The findings of the study will be disseminated to the participants and their guardians.

\section{Statistical analysis}

The statistical analysis will be performed using SPSS V.22.0 software (IBM). Baseline values of demographic differences in interventional groups and the control group will be examined using $\chi^{2}$ tests. The primary and secondary outcomes over time (ie, baseline, 12 weeks, after 12-week follow-up) will be analysed by mixed-design repeated measures ANOVA for between/within-group differences. Bonferroni's post hoc test will be applied to compare results where main effects are significant. Data will be expressed as the mean and SD or SE, and significance will be set at $\mathrm{p}<0.05$. An intention-to-treat analysis will be adopted to deal with missing data, including all participants in the analysis based on the initial group allocation. A linear mixed model approach (a direct likelihood estimation method) will be applied to analyse all continuous variables as value is missing at random. All analyses will include H\&Y and Montreal Cognitive Assessment as covariates, as these variables may differ significantly between groups.

\section{DISCUSSION}

Medical treatment integrated with exercise therapy is still an indispensable method to manage the motor dysfunction for PD. FOG is common in people with PD which contributes to a protective postural response impairment and increases the risk of falls. Nevertheless, this balance instability can be improved by extra training. ${ }^{39}$ Qigong exercise synthesises training in balance, flexibility, neuromuscular coordination and cognition. It consists of body consciousness, attention, imagination, multiple activities and goal-oriented training which may benefit the improvement of gait and postural control, beyond conventional single-mode exercise. ${ }^{40}$

Patients with PD exhibit difficulty in transitions from static to dynamic states. Transitional activity is a vital component of physical activities, especially gait initiation, turning and gait termination. Due to the deficiency in postural control, patients generate excessive trunk movement that causes swaying beyond the limits of stability. ${ }^{41}$ Moreover, people with PD often manifest impaired protective postural response and postural adjustment in preparation for stepping, thus increasing the risk of fall. ${ }^{39}$ Qigong practice requires that the centre of gravity moves and changes to accompany with the movement of the upper limb, slow movement, body control in space and shifting body weight in different directions. Hence, Qigong practice can be beneficial to improve the ability to focus on the base of support and postural stability, as well as enhance core muscle to stress weight-bearing joints and to increase proprioception input of trunk and lower limb joints.

Most Qigong movements involve closed-chain exercise of the lower limbs, and contribute to rectifying deficiencies of heel stride and knee extension on gait cycle. ${ }^{14} 40$ In addition, the meditative movement in Qigong exercise can relieve psychological load and consumption, which may benefit for practitioner to reduce muscle tension, and alleviate effect of freezing on stepping forward.$^{43}$ Furthermore, Qigong is characterised by slow movement incorporated with moderated breathing, as well as keeping 
Table 2 Changes over time within and between control and experimental groups

\begin{tabular}{|c|c|c|c|c|c|c|c|c|}
\hline Group & Baseline* $^{*}$ & 12 weeks* $^{*}$ & Follow-up* & $\begin{array}{l}\text { Mean } \\
\text { difference } \\
\text { at } \\
12 \text { weekst }\end{array}$ & $\begin{array}{l}\text { Mean } \\
\text { difference at } \\
\text { follow-up }\end{array}$ & $\begin{array}{l}\boldsymbol{F}(\mathbf{P} \\
\text { value) } \\
\text { Time } \\
\text { effect }\end{array}$ & $\begin{array}{l}F \text { (P } \\
\text { value) } \\
\text { Group } \\
\text { effect }\end{array}$ & $\begin{array}{l}F \text { (P value) } \\
\text { Interaction } \\
\text { effect }\end{array}$ \\
\hline \multicolumn{9}{|l|}{ Primary outcome } \\
\hline \multicolumn{9}{|l|}{ Stride length (cm) } \\
\hline \multicolumn{9}{|l|}{ Integrated Qigong } \\
\hline \multicolumn{9}{|l|}{ Control } \\
\hline \multicolumn{9}{|l|}{ Gait velocity $(\mathrm{cm} / \mathrm{s})$} \\
\hline \multicolumn{9}{|l|}{ Integrated Qigong } \\
\hline \multicolumn{9}{|l|}{ Balance training } \\
\hline \multicolumn{9}{|l|}{ Integrated Qigong } \\
\hline \multicolumn{9}{|l|}{ Balance training } \\
\hline \multicolumn{9}{|l|}{ Control } \\
\hline \multicolumn{9}{|l|}{$\begin{array}{l}\text { Stride time } \\
\text { variability }(\mathrm{CV})\end{array}$} \\
\hline \multicolumn{9}{|l|}{ Integrated Qigong } \\
\hline \multicolumn{9}{|l|}{ Balance training } \\
\hline \multicolumn{9}{|l|}{ Control } \\
\hline \multicolumn{9}{|l|}{ NFOGQ } \\
\hline \multicolumn{9}{|l|}{ FGA } \\
\hline Balance training & & & & & & & & \\
\hline Control & & & & & & & & \\
\hline MDS-UPDRS Part III & & & & & & & & \\
\hline Integrated Qigong & & & & & & & & \\
\hline Balance training & & & & & & & & \\
\hline Control & & & & & & & & \\
\hline Mini-BESTest & & & & & & & & \\
\hline Integrated Qigong & & & & & & & & \\
\hline Balance training & & & & & & & & \\
\hline Control & & & & & & & & \\
\hline Total falls, $n$ & & & & & & & & \\
\hline Integrated Qigong & & & & & & & & \\
\hline Balance training & & & & & & & & \\
\hline Control & & & & & & & & \\
\hline MFES & & & & & & & & \\
\hline Integrated Qigong & & & & & & & & \\
\hline Balance training & & & & & & & & \\
\hline
\end{tabular}


Table 2 Continued

\begin{tabular}{|c|c|c|c|c|c|c|c|c|}
\hline Group & Baseline* & 12 weeks* & Follow-up* & $\begin{array}{l}\text { Mean } \\
\text { difference } \\
\text { at } \\
12 \text { weeks }\end{array}$ & $\begin{array}{l}\text { Mean } \\
\text { difference at } \\
\text { follow-up }\end{array}$ & $\begin{array}{l}\boldsymbol{F} \text { (P } \\
\text { value) } \\
\text { Time } \\
\text { effect }\end{array}$ & $\begin{array}{l}F(P \\
\text { value) } \\
\text { Group } \\
\text { effect }\end{array}$ & $\begin{array}{l}F \text { ( } P \text { value) } \\
\text { Interaction } \\
\text { effect }\end{array}$ \\
\hline
\end{tabular}

Control

PDQ-39

Integrated Qigong

Balance training

Control

${ }^{*}$ Mean values (SD).

†Mean difference (SE).

CV, coefficient of variation; FGA, Functional Gait Assessment; MDS-UPDRS Part III, Movement Disorder Society Unified Parkinson's Disease

Rating Scale, motor subscale; MFES, Modified Falls Efficacy Scale; Mini-BESTest, Mini-Balance Evaluation Systems Test; NFOGQ, New

Freezing of Gait Questionnaire; PDQ-39, 39-item Parkinson's Disease Questionnaire.

the mind in a state of calm relaxation. The intensity of Qigong is around 1.5-2.6 metabolic equivalents and the average induced maximum heart rate ranges from $43 \%$ to $49 \%$ of the predicted maximum. ${ }^{13}$ Therefore, Qigong is a low-intensity physical exercise that has lower risk of muscle strain and overfatigue and is suitable for individuals with PD as a long-term physical exercise programme.

There are several limitations in this study. First, it is difficult to achieve a blinded intervention for this study because exercise that served as an intervention is widely open to the participants. Second, the control group will have much less contact than training groups, this may induce bias to the study. In the future, the impact of attention/social interaction for each participant would be controlled to ensure the equal dosage of training and attention in different interventions. Third, participants are limited to people with mild to moderate PD. Therefore, it is unclear whether the results would be valid for people with advanced PD. Fourth, the study patients will come from the same geographic area which limits the generalisability and so that it is not a multicentre trial.

For a variety of features of gait pattern, whatever muscle stiffness or abnormal postural control, we may consider the global or specific efficacy of Qigong on gait function in PD. ${ }^{44}$ We expect that a 12 -week Integrated Qigong exercise can establish positive therapeutic effects on patients with PD who have FOG. However, gait deficiency in individuals with PD is a complex syndrome and is associated with neural control regulated by different areas of the brain, particularly the prefrontal lobe and related circuits. ${ }^{45}$ In future studies, we might focus on the mechanism of the different forms of Qigong on the relationship between brain activities and FOG, and explore whether Qigong exercise is conducive to restoring brain function and/or preventing brain degeneration. We hope this trial will demonstrate that the Integrated Qigong exercise promotes the recovery of gait function and prevention of falls for people with mild and moderate PD. The results of this study may furnish evidence to support the beneficial effects of Qigong exercise on improvement of walking ability and reduction of fall risk in people with PD. The findings of this study will provide evidence for a supplemental therapy to manage gait disorder for clinicians and physical therapists.

Acknowledgements The authors thank Fuzhong Li (a professor of Oregon Research Institute) for offering advice for the article framework. The authors also thank the patient advisors and all the participants for their advice and support for the programme.

Contributors ZL, JZ and ZW conceived the conception and design of the trial, and drafted the manuscript. GX, KJ and WY participated in trial registration, communication and monitoring. YJ and TW carried out statistical calculations. YZ provided medical clearance and Parkinson's disease stage diagnoses for the participating patients. All authors participated in revision of the manuscript and approved the final version.

Funding This research was supported by the General Administration of Sport of China Technology Services Project (2017B016), the National Key R\&D Program of China (2017YFC1310300) and the Shanghai Key Lab of Human Performance (Shanghai University of Sport) (11DZ2261100).

Competing interests None declared.

Patient consent for publication Obtained.

Ethics approval This work was approved by the Ethics Committee of Science Research of Shanghai University of Sport (protocol number: 2018031).

Provenance and peer review Not commissioned; externally peer reviewed.

Open access This is an open access article distributed in accordance with the Creative Commons Attribution Non Commercial (CC BY-NC 4.0) license, which permits others to distribute, remix, adapt, build upon this work non-commercially, and license their derivative works on different terms, provided the original work is properly cited, appropriate credit is given, any changes made indicated, and the use is non-commercial. See: http://creativecommons.org/licenses/by-nc/4.0/.

\section{REFERENCES}

1. Ishii M, Okuyama K. Characteristics associated with freezing of gait in actual daily living in Parkinson's disease. J Phys Ther Sci 2017;29:2151-6.

2. Hely MA, Reid WGJ, Adena MA, et al. The Sydney multicenter study of Parkinson's disease: the inevitability of dementia at 20 years. Mov Disord 2008;23:837-44.

3. Nieuwboer A, Giladi N. Characterizing freezing of gait in Parkinson's disease: models of an episodic phenomenon. Mov Disord 2013;28:1509-19.

4. Ehgoetz Martens KA, Lukasik EL, Georgiades MJ, et al. Predicting the onset of freezing of gait: a longitudinal study. Mov Disord 2018;33:128-35 
5. Gilat M, Lígia Silva de Lima A, Bloem BR, et al. Freezing of gait: promising avenues for future treatment. Parkinsonism Relat Disord 2018;52:7-16.

6. Nutt JG, Bloem BR, Giladi N, et al. Freezing of gait: moving forward on a mysterious clinical phenomenon. Lancet Neurol 2011;10:734-44.

7. Schlenstedt C, Shalash A, Muthuraman M, et al. Effect of highfrequency subthalamic neurostimulation on gait and freezing of gait in Parkinson's disease: a systematic review and meta-analysis. Eur $J$ Neurol 2017;24:18-26.

8. Ferrazzoli D, Ortelli P, Zivi I, et al. Efficacy of intensive multidisciplinary rehabilitation in Parkinson's disease: a randomised controlled study. J Neurol Neurosurg Psychiatry 2018;89:828-35.

9. Fox SH, Katzenschlager R, Lim S-Y, et al. International Parkinson and movement disorder society evidence-based medicine review: update on treatments for the motor symptoms of Parkinson's disease. Mov Disord 2018;33:1248-66.

10. Klich W, Milert A. Tai chi and Qigong as a form of physical activity of people of all ages in the context of modern physiotherapy. Phys. Act. Rev. 2018;6:22-8.

11. Chang P-S, Knobf MT, Oh B, et al. Physical and psychological effects of Qigong exercise in community-dwelling older adults: an exploratory study. Geriatr Nurs 2018:39:88-94.

12. Chen S, Zhang Y, Wang YT, et al. Traditional Chinese mind and body exercises for promoting balance ability of old adults: a systematic review and meta-analysis. Evid Based Complement Alternat Med 2016;2016:1-9

13. Xiao C-M, Zhuang Y-C. Effect of health Baduanjin Qigong for mild to moderate Parkinson's disease. Geriatr Gerontol Int 2016;16:911-9.

14. Liu XL, Chen S, Wang Y. Effects of health Qigong exercises on relieving symptoms of Parkinson's disease. Evid Based Complement Alternat Med 2016;2016:5935782:1-11.

15. Lin C-Y, Wei T-T, Wang C-C, et al. Acute physiological and psychological effects of Qigong exercise in older practitioners. Evid Based Complement Alternat Med 2018;2018:1-10.

16. Zhang F, Bai Y-H, Zhang J. The Influence of "wuqinxi" exercises on the Lumbosacral Multifidus. J Phys Ther Sci 2014;26:881-4.

17. Guo Y, Xu M, Wei Z, et al. Beneficial Effects of Qigong Wuqinxi in the Improvement of Health Condition, Prevention, and Treatment of Chronic Diseases: Evidence from a Systematic Review. Evid Based Complement Alternat Med 2018;2018:1-40.

18. Li M, Fang Q, Li J, et al. The effect of Chinese traditional exercise-Baduanjin on physical and psychological well-being of college students: a randomized controlled trial. PLOS One 2015;10:e0130544.

19. Ge L, Zheng Q-X, Liao Y-T, et al. Effects of traditional Chinese exercises on the rehabilitation of limb function among stroke patients: a systematic review and meta-analysis. Complement Ther Clin Pract 2017;29:35-47.

20. Wu W, Liu X, Liu J, et al. Effectiveness of water-based Liuzijue exercise on respiratory muscle strength and peripheral skeletal muscle function in patients with COPD. Int $J$ Chron Obstruct Pulmon Dis 2018;13:1713-26.

21. Cohen J. Statistical power analysis for the behavioral sciences. 2nd edn. Hillsdale, NJ: Erlbaum, 1988.

22. Chinese Health Qigong Association. Theoretical training course in health Qigong. Beijing, CHN: Foreign Languages Press Co. Ltd, 2015.

23. Atterbury EM, Welman KE. Balance training in individuals with Parkinson's disease: Therapist-supervised vs. home-based exercise programme. Gait Posture 2017;55:138-44.

24. Delval A, Snijders AH, Weerdesteyn V, et al. Objective detection of subtle freezing of gait episodes in Parkinson's disease. Mov Disord 2010;25:1684-93.

25. Lin C-C, Wagenaar RC. The impact of walking speed on interlimb coordination in individuals with Parkinson's disease. J Phys Ther Sci 2018;30:658-62.
26. Lord S, Galna B, Rochester L. Moving forward on gait measurement: toward a more refined approach. Mov Disord 2013;28:1534-43.

27. Harrison EC, McNeely ME, Earhart GM. The feasibility of singing to improve gait in Parkinson disease. Gait Posture 2017;53:224-9.

28. Nieuwboer A, Rochester L, Herman T, et al. Reliability of the new freezing of gait questionnaire: agreement between patients with Parkinson's disease and their carers. Gait Posture 2009;30:459-63.

29. Beninato $\mathrm{M}$, Ludlow $\mathrm{LH}$. The functional gait assessment in older adults: validation through Rasch modeling. Phys Ther 2016;96:456-68

30. Leddy AL, Crowner BE, Earhart GM. Functional gait assessment and balance evaluation system test: reliability, validity, sensitivity, and specificity for identifying individuals with Parkinson disease who fall. Phys Ther 2011;91:102-13.

31. Yang $Y$, Wang $Y$, Zhou $Y$, et al. Validity of the functional gait assessment in patients with Parkinson disease: construct, concurrent, and predictive validity. Phys Ther 2014;94:392-400.

32. Mak MKY, Auyeung MM. The mini-BESTest can predict parkinsonian recurrent fallers: a 6-month prospective study. J Rehabil Med 2013;45:565-71.

33. Benka Wallén M, Sorjonen K, Löfgren N, et al. Structural validity of the Mini-Balance evaluation systems test (Mini-BESTest) in people with mild to moderate Parkinson disease. Phys Ther 2016;96:1799-806.

34. Strouwen C, Molenaar EALM, Münks L, et al. Training dual tasks together or apart in Parkinson's disease: results from the duality trial. Mov Disord 2017;32:1201-10.

35. O'Halloran AM, Pénard N, Galli A, et al. Falls and falls efficacy: the role of sustained attention in older adults. BMC Geriatr 2011;11:85

36. Pua Y-H, Ong P-H, Clark RA, et al. Falls efficacy, postural balance, and risk for falls in older adults with falls-related emergency department visits: prospective cohort study. BMC Geriatr 2017;17:291.

37. Tu X-J, Hwang W-J, Hsu S-P, et al. Responsiveness of the shortform health survey and the Parkinson's disease questionnaire in patients with Parkinson's disease. Health Qual Life Outcomes 2017;15:75.

38. Jesus-Ribeiro J, Vieira E, Ferreira P, et al. Reliability and validity of 39-Item Parkinson's disease questionnaire and Parkinson's disease quality of life questionnaire. Acta Med Port 2017;30:395-401.

39. Peterson DS, Horak FB. Effects of freezing of gait on postural motor learning in people with Parkinson's disease. Neuroscience 2016;334:283-9.

40. Klein P, Picard G, Baumgarden J, et al. Meditative movement, energetic, and physical analyses of three Qigong exercises: unification of eastern and Western mechanistic exercise theory. Medicines 2017:4.

41. Bovonsunthonchai S, Vachalathiti R, Pisarnpong A, et al. Spatiotemporal gait parameters for patients with Parkinson's disease compared with normal individuals. Physiother Res Int 2014;19:158-65.

42. Okuma Y. Freezing of gait and falls in Parkinson's disease. $J$ Parkinsons Dis 2014;4:255-60.

43. Liu X-Y, Gao J, Yin B-X, et al. Efficacy of Ba Duan Jin in improving balance: a study in Chinese community-dwelling older adults. $J$ Gerontol Nurs 2016;42:38-46.

44. Sawynok J, Lynch M. Qigong and fibromyalgia: randomized controlled trials and beyond. Evid Based Complement Alternat Med 2014;2014:1-14.

45. Maidan I, Nieuwhof F, Bernad-Elazari $\mathrm{H}$, et al. The role of the fronta lobe in complex walking among patients with Parkinson's disease and healthy older adults: an fNIRS study. Neurorehabil Neural Repair 2016;30:963-71.

46. Washburn RA, McAuley E, Katula J, et al. The physical activity scale for the elderly (PASE): evidence for validity. J Clin Epidemiol 1999;52:643-51. 